\title{
Hot and cool water in Herbig Ae protoplanetary disks
}

\section{A challenge for Herschel}

\author{
P. Woitke ${ }^{1,2}$, W.-F. Thi ${ }^{3}$, I. Kamp ${ }^{4}$, and M. R. Hogerheijde ${ }^{5}$ \\ 1 UK Astronomy Technology Centre, Royal Observatory, Edinburgh, Blackford Hill, Edinburgh EH9 3HJ, UK \\ e-mail: ptw@roe.ac.uk \\ 2 School of Physics \& Astronomy, University of St. Andrews, North Haugh, St. Andrews KY16 9SS, UK \\ 3 SUPA, Institute for Astronomy, University of Edinburgh, Royal Observatory, Blackford Hill, Edinburgh EH9 3HJ, UK \\ 4 Kapteyn Astronomical Institute, Postbus 800, 9700 AV Groningen, The Netherlands \\ 5 Leiden Observatory, Leiden University, PO Box 9513, 2300 RA Leiden, The Netherlands \\ Received 1 April 2009 / Accepted 30 May 2009
}

\section{ABSTRACT}

\begin{abstract}
The spatial origin and detectability of rotational $\mathrm{H}_{2} \mathrm{O}$ emission lines from Herbig Ae type protoplanetary disks beyond $70 \mu \mathrm{m}$ is discussed. We use the recently developed disk code PRODIMO to calculate the thermo-chemical structure of a Herbig Ae type disk and apply the non-LTE line radiative transfer code RATRAN to predict water line profiles and intensity maps. The model shows three spatially distinct regions in the disk where water concentrations are high, related to different chemical pathways to form the water: (1) a big water reservoir in the deep midplane behind the inner rim, (2) a belt of cold water around the distant icy midplane beyond the "snowline" $r \gtrsim 20 \mathrm{AU}$, and (3) a layer of irradiated hot water at high altitudes $z / r=0.1 \ldots 0.3$, extending from about $1 \mathrm{AU}$ to $30 \mathrm{AU}$, where the kinetic gas temperature ranges from $200 \mathrm{~K}$ to $1500 \mathrm{~K}$. Although region 3 contains only little amounts of water vapour $\left(\sim 10^{-4} M_{\text {Earth }}\right)$, it is this warm layer that is almost entirely responsible for the rotational water emission lines as observable with Herschel. Only one ortho and two para $\mathrm{H}_{2} \mathrm{O}$ lines with the lowest excitation energies $<100 \mathrm{~K}$ are found to originate partly from region 2. We conclude that observations of rotational water lines from Herbig Ae disks probe first and foremost the conditions in region 3, where water is predominantly formed via neutral-neutral reactions and the gas is thermally decoupled from the dust $T_{\text {gas }}>T_{\text {dust }}$. The observation of rotational water lines does not allow for a determination of the snowline, because the snowline truncates the radial extension of region 1, whereas the lines originate from the region 3. Different line transfer approximations (LTE, escape probability, Monte Carlo) are discussed. A non-LTE treatment is required in most cases, and the results obtained with the escape probability method are found to underestimate the Monte Carlo results by $2 \%-45 \%$.
\end{abstract}

Key words. astrochemistry - circumstellar matter - stars: formation - radiative transfer - methods: numerical

\section{Introduction}

Water is one of the most important species in planet formation, disk evolution and for the origin of life. In protoplanetary disks, water can be abundant either in the gas phase or as solid ice, owing to a high sublimation temperature.

Water vapour is predicted to be abundant inside of the snowline within a few AU where densities are high and temperatures are too warm for ice formation (Agúndez et al. 2008; Glassgold et al. 2009). Indeed, observations in the near- and mid-infrared with Spitzer (Salyk et al. 2008; Carr \& Najita 2008; Najita et al. 2009; Eisner et al. 2009) reveal the presence of water vapour and other simple organic molecules $\left(\mathrm{OH}, \mathrm{C}_{2} \mathrm{H}_{2}, \mathrm{HCN}, \mathrm{CO}_{2}\right)$. Simple modelling of the line emissions constrains the warm gas (a few $100 \mathrm{~K}$ ) to be located inside of $r \lesssim 3-5 \mathrm{AU}$.

The core-accretion model for planet formation is only efficient in the cold midplane of disks where dust grains are covered by water ice. Icy grains are stickier than bare silicate grains and coagulate faster into planetesimals (Ida \& Lin 2008). Liquid water is one of the prerequisites for the emergence of life on terrestrial planets. But its origin, either via release of water molecules trapped in hydrated rocks during volcanism or via the impact of comets, is still being debated (Nuth 2008).

The lowest rotational lines of water lie in the far IR and are only observable by satellites, e.g. the Herschel Space Observatory. Other recent works on rotational water lines from protoplanetary disks used X-ray models (without UV photoprocesses) to calculate the thermo-chemical disk structure on top of a pre-described density structure (Meijerink et al. 2008) and applied a multi-zone escape probability method (Poelman \& Spaans 2005, 2006) to compute the line fluxes. In this Letter, we use the disk code PRODIMO to compute the disk structure, temperature, and water abundance self-consistently, and discuss the prospects for detecting rotational water lines by means of the Monte Carlo code RATRAN (Hogerheijde \& van der Tak 2000).

\section{The model}

We used the recently developed disk code PRODIMO to calculate the thermo-chemical structure of a protoplanetary disk around a Herbig Ae type star with parameters listed in Table 1. PRODIMO combines frequency-dependent 2D dust-continuum radiative transfer, kinetic gas-phase and UV photo-chemistry, ice formation, and detailed non-LTE heating \& cooling with the consistent calculation of the hydrostatic disk structure. PRODIMO does not include X-rays at the moment. X-ray to FUV luminosity ratios of Herbig Ae stars are often low $L_{\mathrm{X}} / L_{\mathrm{FUV}} \ll 1$ (Kamp et al. 2008), so that we assume that the FUV irradiation provides the main energy input for the disk. The model is characterised by a high degree of consistency between the various physical, chemical, and radiative processes, where the mutual feedbacks are solved by global iterations. For more details see Woitke et al. 
Table 1. Herbig Ae type disk model parameter.

\begin{tabular}{lcc}
\hline \hline Quantity & Symbol & Value \\
\hline stellar mass & $M_{\star}$ & $2.2 M_{\odot}$ \\
effective temperature & $T_{\text {eff }}$ & $8600 \mathrm{~K}$ \\
stellar luminosity & $L_{\star}$ & $32 L_{\odot}$ \\
\hline disk mass & $M_{\text {disk }}$ & $0.01 M_{\odot}$ \\
inner disk radius & $R_{\text {in }}$ & $0.5 \mathrm{AU}^{(1)}$ \\
outer disk radius & $R_{\text {out }}$ & $150 \mathrm{AU}$ \\
radial column density power index & $\epsilon$ & 1.0 \\
\hline dust-to-gas mass ratio & $\rho_{d} / \rho$ & 0.01 \\
minimum dust particle radius & $a_{\text {min }}$ & $0.1 \mu \mathrm{m}$ \\
maximum dust particle radius & $a_{\max }$ & $200 \mu \mathrm{m}$ \\
dust size distribution power index & $a_{\mathrm{pow}}$ & 3.5 \\
dust material mass density $(2)$ & $\rho_{\mathrm{gr}}$ & $2.5 \mathrm{~g} \mathrm{~cm}^{-3}$ \\
\hline strength of incident ISM UV & $\chi^{\mathrm{ISM}}$ & 1 \\
abundance of PAHs relative to ISM & $f_{\mathrm{PAH}}$ & 0.1 \\
\hline
\end{tabular}

(1) Soft inner edge applied, see Woitke et al. (2009); (2): dust optical constants from Draine \& Lee (1984).

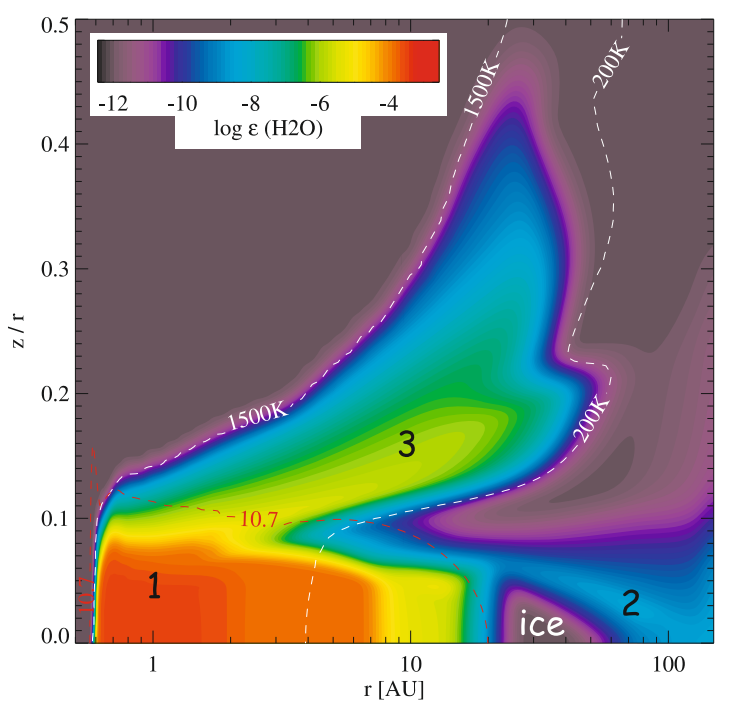

Fig. 1. Concentration of water molecules $\epsilon_{\mathrm{H}_{2} \mathrm{O}}=n_{\mathrm{H}_{2} \mathrm{O}} / n_{\langle\mathrm{H}\rangle}$ as function of radius $r$ and relative height above the midplane $z / r$ for a Herbig Ae disk model. Three different regions with high water concentration can be distinguished as described in the text. The two white contours show $T_{\text {gas }}=200 \mathrm{~K}$ and $1500 \mathrm{~K}$. The red contour line marks total hydrogen nuclei particle density $n_{\langle\mathrm{H}\rangle}=10^{10.7} \mathrm{~cm}^{-3}$.

(2009), henceforth called Paper I. Recent updates include an improved treatment of UV photorates by detailed cross sections in the calculated radiation field (see Kamp et al. 2009).

The model results in a flared disk structure with a puffedup inner rim and a vertically extended hot atomic layer above $z / r \gtrsim 0.15-0.4$ from the inner rim to about $r=20 \mathrm{AU}$, similar to Fig. 9 in Paper I (1.h.s.), where $T_{\text {gas }}>1000 \mathrm{~K}$ due to the stellar UV-irradiation. The major difference between the T Tauri type disk discussed in Paper I and the Herbig Ae disk discussed here is that in Herbig disks, the star is much more luminous, so the dust is warmer in the midplane (here $T_{\text {dust }}>100 \mathrm{~K}$ inward of $r=20 \mathrm{AU}$ ), which prevents water ice formation.

Water molecules generally form in deeper layers, and the resulting water concentration in these layers is depicted in Fig. 1. The vertical $\mathrm{H}_{2} \mathrm{O}$ column densities in this model are found to be $10^{22} \mathrm{~cm}^{-2}$ at $1 \mathrm{AU}$, still $10^{19} \mathrm{~cm}^{-2}$ at $10 \mathrm{AU}$, but then quickly dropping below $10^{15} \mathrm{~cm}^{-2}$ at $30 \mathrm{AU}$ and beyond.
Table 2. Characteristics of water regions in Herbig Ae disk model.

\begin{tabular}{|c|c|c|c|c|c|}
\hline Region & $\overline{c r[\mathrm{AU}]}$ & $z / r$ & $\overline{M_{\mathrm{H}_{2} \mathrm{O}}\left[M_{\text {Earth }}\right.}$ & $\left\langle\overline{\left\langle T_{\text {gas }}\right\rangle[\mathrm{K}]}\right.$ & $\overline{\left\langle\left\langle T_{\text {dust }}\right\rangle[\mathrm{K}]\right.}$ \\
\hline 1 & $0.7-20$ & $<0.1$ & 0.31 & 240 & 240 \\
\hline 2 & $20-150$ & $<0.1$ & $3.2 \times 10^{-5}$ & 38 & 37 \\
\hline 3 & $1-30$ & $0.1-0.3$ & $1.1 \times 10^{-4}$ & 410 & 150 \\
\hline
\end{tabular}

\section{Chemical pathways to water}

The formation of $\mathrm{H}_{2} \mathrm{O}$ follows different chemical pathways in the three different regions shown in Fig. 1. Two of these regions ( 1 and 3 ) have been previously identified in vertical slab models for X-ray irradiated T Tauri disks by Glassgold et al. (2009).

1) The big inner water reservoir. The deep midplane regions from just behind the inner rim to a distance of about $10 \mathrm{AU}$ in this model host the majority of the water in the disk, see Table 2. This region is almost completely shielded from the stellar and interstellar radiation $\left(A_{V}>10\right)$, is still too warm for water to freeze out $\left(T_{\text {dust }}>120 \mathrm{~K}\right)$, and is characterised by large particle densities $>10^{11} \mathrm{~cm}^{-3}$ and thermal equilibrium between gas and dust ( $T_{\text {gas }} \approx T_{\text {dust }}$ ). The important chemical reactions in this region are in detailed balance with their direct reverse reaction; i.e. the gas-phase chemistry is close to thermochemical equilibrium. Since $\mathrm{H}_{2} \mathrm{O}$ is the thermodynamically most stable oxygen carrier under these conditions (after $\mathrm{CO}$ ), the water concentration is given approximately by the element abundance of oxygen minus the fraction thereof bound in CO.

2) The distant water belt. Region 2 at $r \approx 20-150 \mathrm{AU}$ and $z / r \lesssim 0.05$ is characterised by particle densities $\sim 10^{9}-10^{10} \mathrm{~cm}^{-3}$, temperatures $T_{\text {gas }} \approx T_{\text {dust }} \approx 30-100 \mathrm{~K}$, and UV-strengths $\chi$ (see Eq. (41) in Paper I) below 500. These conditions allow water to freeze out, and there is a tight equilibrium between water adsorption and photodesorption $\mathrm{H}_{2} \mathrm{O} \leftrightarrow \mathrm{H}_{2} \mathrm{O}^{\#}$, where $\mathrm{H}_{2} \mathrm{O}^{\#}$ designates water ice. The formation of gaseous water in this region is mainly controlled by the following two photoreactions

$$
\begin{aligned}
\mathrm{H}_{2} \mathrm{O}^{\#}+\mathrm{h} v & \longrightarrow \mathrm{H}_{2} \mathrm{O} \\
\mathrm{H}_{2} \mathrm{O}+\mathrm{h} v & \longrightarrow \mathrm{OH}+\mathrm{H} .
\end{aligned}
$$

Therefore, the strength of the UV field $\chi$ controls the water vapour concentration ${ }^{1}$. At $r=80 \mathrm{AU}, \chi$ drops from $\sim 10^{3}$ to virtually 0 between $z / r \approx 0.15$ and 0.02 . The UV photons mostly come from the star and are scattered around the optically thick core of the disk. Water is photo-dissociated for $\chi$ that is too high, and freezes out for too low $\chi$. The result is a thin belt of cold water vapour with concentrations in excess of $10^{-9}$ around the distant icy midplane. Glassgold et al. (2009) have not found region 2 in their T Tauri models, probably because their model did not include the UV photoprocesses.

3) The hot water layer. At distances $r \approx 1-30 \mathrm{AU}$ and relative heights $z / r \lessgtr 0.1-0.3$, the model is featured by an additional layer of warm water-rich gas that is thermally decoupled from the dust $T_{\text {gas }}>T_{\text {dust }}$. The particle density is about $\sim 10^{8}-10^{10} \mathrm{~cm}^{-3}$ in this layer, the dust temperature $100-200 \mathrm{~K}$, the gas temperature $200-1500 \mathrm{~K}$, and the UV radiation field strength $\chi \approx 10^{4}-10^{7}$.

Above region 3, the high gas temperatures in combination with the direct UV irradiation from the star efficiently destroys

\footnotetext{
1 Our analysis is restricted to the case of kinetic chemical equilibrium. Thus, we cannot discuss the history of water ice formation with our model. Water ice may furthermore be photodesorbed directly into $\mathrm{OH}$, see e.g. Andersson \& van Dishoeck (2008).
} 

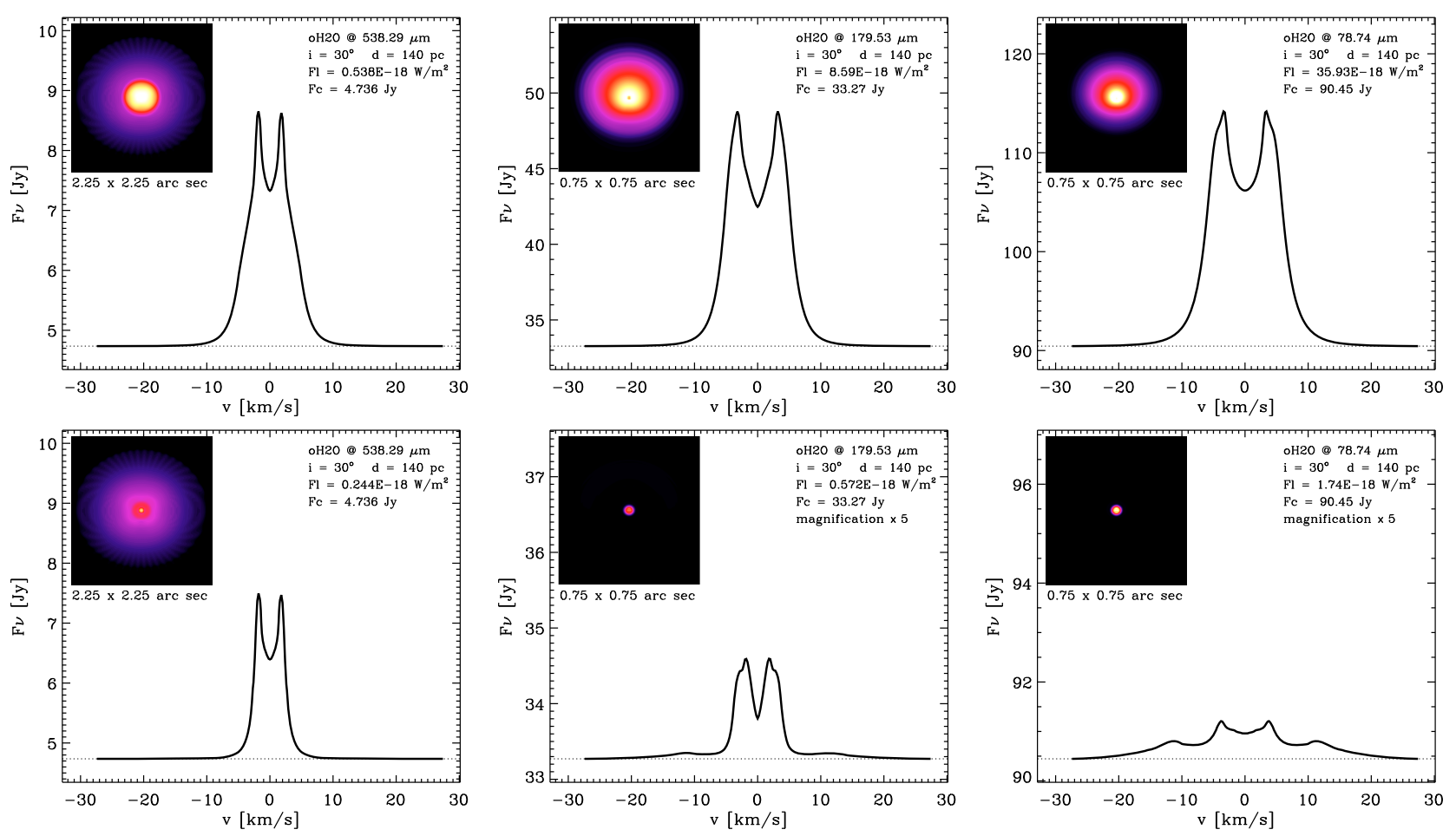

Fig. 2. Monte Carlo simulations of three ortho $\mathrm{H}_{2} \mathrm{O}$ lines with increasing excitation energy for a distance of $140 \mathrm{pc}$ and inclination $30^{\circ}$ (infinite resolution). The inserted maps show the integrated line intensity $I(x, y)=\int I_{v}(x, y)-I_{v}^{\text {cont }}(x, y) \mathrm{d} v$ as function of sky position $(x, y)$ with $I_{v}^{\text {cont }}(x, y)=$ $I_{v}(x, y)$ far away from line centre. An angular distance of $0.3^{\prime \prime}$ in the maps corresponds to a spatial distance of 42 AU. The first row shows the results for the full thermo-chemical input model. The second row shows the results with the water molecules being removed from region 3 (see Fig. 1). The low-excitation line on the 1.h.s. is depicted on a $3 \times$ larger angular scale. The scaling of the spectral flux $F_{v}$ is partly enlarged by a factor $\times 5$ in the lower panels as indicated. The colour-scale for the intensity maps is the same in each column.

all $\mathrm{OH}$ and $\mathrm{H}_{2} \mathrm{O}$. In region 3 , the medium is shielded from the direct stellar irradiation by the puffed-up inner rim. In the shadow of the inner rim, $\chi$ drops quickly by about two orders of magnitude (the remaining UV photons are scattered stellar photons), and water forms via the following chain of surface and neutralneutral reactions

$$
\begin{aligned}
\mathrm{H}+\mathrm{H}+\text { dust } & \longrightarrow \mathrm{H}_{2}+\text { dust } \\
\mathrm{H}_{2}+\mathrm{O} & \longrightarrow \mathrm{OH}+\mathrm{H} \\
\mathrm{OH}+\mathrm{H}_{2} & \longrightarrow \mathrm{H}_{2} \mathrm{O}+\mathrm{H},
\end{aligned}
$$

counterbalanced by the photo-dissociation reactions $\mathrm{OH}+\mathrm{hv} \rightarrow$ $\mathrm{O}+\mathrm{H}$ and $\mathrm{H}_{2} \mathrm{O}+\mathrm{hv} \rightarrow \mathrm{OH}+\mathrm{H}$. The reason for the minimum of the $\mathrm{H}_{2} \mathrm{O}$ concentration between regions 3 and 1 lies in the high activation energies required for reactions (4) and (5). These neutral-neutral reactions become inefficient when the gas temperature drops below $T_{\text {gas }} \lesssim 200 \mathrm{~K}$. The radial extension of the hot water layer is restricted for the same reason. Since UV chemistry is lacking in (Glassgold et al. 2009), their water formation is counterbalanced by charge transfer reactions with $\mathrm{H}+$, which also destroys $\mathrm{OH}$ and $\mathrm{H}_{2} \mathrm{O}$.

\section{Spectral appearance of rotational water lines}

Having calculated the density structure, the molecular abundances, the dust and gas temperatures and the continuous radiation field in the disk, we performed axisymmetric non-LTE line transfer calculations for selected rotational water lines as summarised in Table 3. The non-LTE input data for ortho (para) $\mathrm{H}_{2} \mathrm{O}$ is taken from the Leiden LAMBDA database (Schöier et al. 2005), which includes 45 (45) levels, 158 (157) lines, and 990 (990) collisional transitions with $\mathrm{H}_{2}$. A scaled version of the last data is

\begin{tabular}{|c|c|c|c|c|c|c|c|}
\hline Carrier & Transition $\lambda[\mu \mathrm{m}]$ & $A_{\mathrm{ul}}\left[\mathrm{s}^{-1}\right]$ & $E_{u}[\mathrm{~K}]$ & LTE & ES & $\mathrm{MC}$ & $10 \mathrm{~T}$ \\
\hline $\mathrm{O}-\mathrm{H}_{2} \mathrm{O}$ & $1_{10} \rightarrow 1_{01} \quad 538.29$ & 0.0035 & 61 & 0.702 & $\overline{0.531}$ & 0.538 & $2 \%$ \\
\hline $\mathrm{H}_{2} \mathrm{O}$ & $4_{23} \rightarrow 3_{12^{\star}} 180$. & & 194 & 27 & 2.32 & 3.39 & $37 \%$ \\
\hline $\mathrm{H}_{2} \mathrm{O}$ & $2_{12} \rightarrow 1_{01} \star 179.53$ & 9 & 114 & 11.9 & 7.69 & 8.59 & $11 \%$ \\
\hline $\mathrm{H}_{2} \mathrm{O}$ & $3_{03} \rightarrow 2_{12} \quad 174.63$ & 0.0505 & 197 & 12.7 & 7.47 & 8.88 & $17 \%$ \\
\hline $\mathrm{I}_{2} \mathrm{O}$ & $2_{12} \rightarrow 1_{10} \quad 108.07$ & 6 & 194 & 54.8 & 11.4 & 17.9 & $44 \%$ \\
\hline $\mathrm{O}-\mathrm{H}_{2} \mathrm{O}$ & $4_{23} \rightarrow 3_{12} \star 78.74$ & 0.484 & 432 & 94.5 & 24.6 & 35.9 & $37 \%$ \\
\hline $\mathrm{p}-\mathrm{H}_{2} \mathrm{O}$ & $2_{02} \rightarrow 1_{11} 303.46$ & 0.0058 & 101 & 1.27 & 1.48 & 1.40 & $5 \%$ \\
\hline${ }_{2} \mathrm{O}$ & $1_{11} \rightarrow 0_{00} \quad 269.27$ & 0.0184 & 53 & 2.40 & 2.22 & 2.09 & $6 \%$ \\
\hline $\mathrm{I}_{2} \mathrm{O}$ & $4_{13} \rightarrow 3_{22}^{\star} 144.52$ & & 397 & 5.53 & 4.57 & 4.15 & $9 \%$ \\
\hline $\mathrm{H}_{2} \mathrm{O}$ & $3_{13} \rightarrow 2_{02} \quad 138.53$ & 0.125 & 204 & 14.3 & 7.76 & 8.71 & $12 \%$ \\
\hline & $2_{20} \rightarrow 1_{11} \quad 100.98$ & 0.260 & 196 & 32.5 & 8.66 & 10.9 & $23 \%$ \\
\hline $\mathrm{p}-\mathrm{H}_{2} \mathrm{O}$ & $3_{22} \rightarrow 2_{11}^{\star} 89.99$ & 0.352 & 297 & 48.0 & 10.2 & 14.0 & $31 \%$ \\
\hline
\end{tabular}

Table 3. Properties of rotational water lines, and calculated line fluxes $F_{L}=\int\left(F_{v}-F_{v}^{\text {cont }}\right) \mathrm{d} v$ for different line transfer methods.

Listed line fluxes are in units $10^{-18} \mathrm{~W} / \mathrm{m}^{2}$ for distance $140 \mathrm{pc}$ and inclination $30^{\circ} .{ }^{\star}$ Indicates that this line will be observed by the Herschel OT Key Programme GASPS. ES = escape probability, MC = Monte Carlo.

also applied to collisions with $\mathrm{H}$, which is essential as region 3 is partly $\mathrm{H}_{2}$-poor and $\mathrm{H}$-rich. The velocity field is assumed to be Keplerian. We add a turbulent line width of $0.15 \mathrm{~km} \mathrm{~s}^{-1}$ to the thermal line width throughout the disk. The ratio between para and ortho $\mathrm{H}_{2} \mathrm{O}$ is assumed to be as in LTE.

We used three different methods of increasing complexity to calculate the water population numbers: local thermal equilibrium (LTE), a simple escape probability method (ES, see Sect. 6.1 of Paper I) and a modified version of the 2D Monte Carlo code RATRAN (Hogerheijde \& van der Tak 2000), see (Kamp et al. 2009) for modifications. The LTE and ES methods used the full $150 \times 150$ PRODIMO output directly as thermochemical input model. The grid size of the MC model needed to 


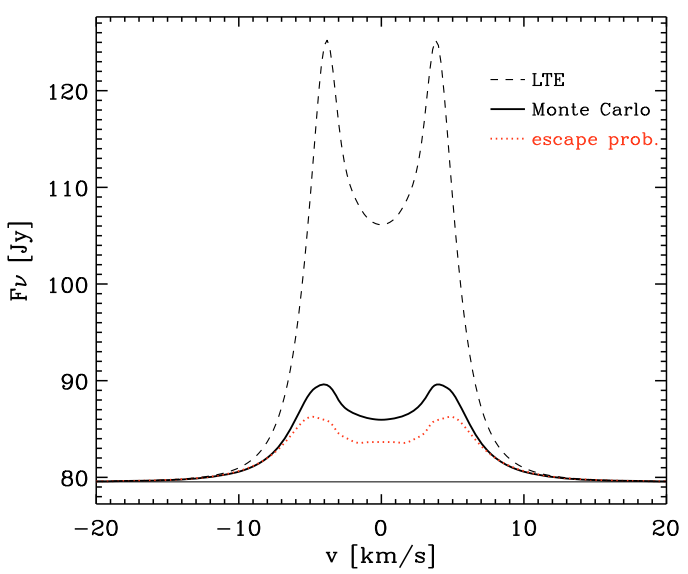

Fig. 3. Comparison between the results of different line transfer methods in application to the high-excitation para- $\mathrm{H}_{2} \mathrm{O}$ line at $89.99 \mu \mathrm{m}$. The full black line redisplays the results of the Monte Carlo treatment already shown in Fig. 2, upper row, middle figure.

be somewhat reduced for practical reasons. We decided to run $80 \times 80$ MC models, which need about $7 \times 10^{5}$ photon packages to converge to a signal/noise ratio better than 5 for the worst population number in the worst cell, which takes about $13 \mathrm{CPU}$ hours on a $2.66 \mathrm{GHz}$ Linux machine.

To investigate the role of the hot water layer (region 3) for the spectral appearance of the rotational lines, we calculated two sets for each model. The first set includes the full chemical input model. In the second set, we artificially put the water abundance in region 3 to zero (if $n_{\langle\mathrm{H}\rangle}<10^{10.7} \mathrm{~cm}^{-3}$ and $T_{\text {gas }}>200 \mathrm{~K}$ ). The results of the two sets of MC models are compared in Fig. 2.

We generally observe double-peaked line profiles typical for rotating gas in emission. In the case of the full input model, the peak separation generally measures the radial extension of region 3 (Kepler velocity is $8.8 \mathrm{~km} \mathrm{~s}^{-1}$ at $25 \mathrm{AU}$ in this model, inclined to $30^{\circ}$ gives $4.4 \mathrm{~km} \mathrm{~s}^{-1}$ ). However, the peak separation of the lowest $\mathrm{o}-\mathrm{H}_{2} \mathrm{O}$ excitation line $(538.29 \mu \mathrm{m})$ and the two lowest p- $\mathrm{H}_{2} \mathrm{O}$ excitation lines (269.27 $\mu \mathrm{m}$ and $303.46 \mu \mathrm{m}$, see Table 3) correspond to the full radial extent of the model, $150 \mathrm{AU}$.

The truncated model generally results in $10-20 \times$ smaller line fluxes with a broader, often unclear profile. If the water in region 3 is missing, the lines originate mainly from region 1 , which is optically thick even in the continuum. Since the gas is in thermal balance with the dust in region $1\left(T_{\text {gas }} \approx T_{\text {dust }}\right)$, the lines do not go much into emission in region 1. Again, the lowest three excitation lines behave differently, and region 2 contributes by $\sim 30 \%$ for these lines. There is furthermore one intermittent case $\left(\mathrm{o}-\mathrm{H}_{2} \mathrm{O} 179.5 \mu \mathrm{m}\right.$ with $\left.E_{u}=114 \mathrm{~K}\right)$ where the truncated model reveals a small contribution of the extended region 2 with the character of low-excitation lines (middle column in Fig. 2).

All rotational water lines of the Herbig Ae disk discussed in this letter are above the $1 \sigma$ detection limit of the PACS spectrometer $\left(\approx 1-5 \times 10^{-18} \mathrm{~W} / \mathrm{m}^{2}\right.$, depending on $\left.\lambda\right)$. The strongest water lines at $78.74 \mu \mathrm{m}$ and $89.99 \mu \mathrm{m}$ are above the $5 \sigma$ detection limit. However, the lines sit on a strong dust continuum, which possibly complicates the detection by Herschel.

\section{LTE vs. escape probability vs. Monte Carlo}

Figure 3 compares the results obtained by three different line transfer methods for the high-excitation para- $\mathrm{H}_{2} \mathrm{O}$ line at $89.99 \mu \mathrm{m}$. The results for the other lines are listed in Table 3 . We consider the deviations from the results of the most advanced method (MC) as a measure of the quality of the other methods. The LTE predictions are generally too high, by up to a factor of 3.5 , although continuum flux, line width, and peak separation are similar. Since the densities in region 3 are lower than the critical density $\sim 10^{10} \mathrm{~cm}^{-3}$, the levels tend to depopulate radiatively, which explains the overpredictions by LTE. Deviations between ES and MC are between $2 \%$ and $45 \%$, and increase with excitation energy $E_{u}$. Our ES method tends to underestimate the line fluxes in general. The levels in region 3 are pumped by line radiation from distant regions in LTE that have larger line source functions. This effect is difficult, if not impossible, to be properly account for in the ES approximation.

\section{Conclusions}

The rotational water lines from Herbig Ae disks beyond $70 \mu \mathrm{m}$ originate predominantly from a warm molecular layer at relative altitudes $z / r \approx 0.1-0.3$ where $\mathrm{H}_{2} \mathrm{O}$ is formed via neutralneutral reactions in a thermally decoupled gas $\left(T_{\text {gas }}>T_{\text {dust }}\right)$. The more distant cold water around the icy midplane, where the ice is photodesorbed, contributes only to the lowest excitation lines. The peak separation of all other lines measures the radial extension of the warm molecular layer, which is about $40 \mathrm{AU}$ in the discussed model. In contrast, the vast majority of water vapour in the disk is situated in the deep midplane, extending from just behind the inner rim outward to the snowline, where water freezes out to form water ice. The gas in this massive deep water reservoir is in thermal balance $\left(T_{\text {gas }} \approx T_{\text {dust }}\right)$ with optically thick dust and, therefore, no strong line emissions are produced with respect to the continuum from this deep region. Thus, no information about the position of the snowline can be deduced from the rotational water lines. A similar conclusion was reached by Meijerink et al. (2008). The line analysis generally requires a non-LTE treatment. Our escape probability method is found to underestimate the water line fluxes with respect to the more expensive Monte Carlo method by about $2 \%-45 \%$.

Acknowledgements. We thank Dr. Rowin Meijerink for an open discussion about water in disks and Dr. Dieter Poelman for internal benchmark tests of different non-LTE line transfer methods.

\section{References}

Agúndez, M., Cernicharo, J., \& Goicoechea, J. R. 2008, A\&A, 483, 831 Andersson, S., \& van Dishoeck, E. F. 2008, A\&A, 491, 907

Carr, J. S., \& Najita, J. R. 2008, Science, 319, 1504

Draine, B. T., \& Lee, H. M. 1984, ApJ, 285, 89

Eisner, J. A., Graham, J. R., Akeson, R. L., \& Najita, J. 2009, ApJ, 692, 309

Glassgold, A. E., Meijerink, R., \& Najita, J. R. 2009

[arXiv: 0905.4523]

Hogerheijde, M. R., \& van der Tak, F. F. S. 2000, A\&A, 362, 697

Ida, S., \& Lin, D. N. C. 2008, ApJ, 685, 584

Kamp, I., Freudling, W., Robberto, M., Chengalur, J., \& Keto, E. 2008, Phys. Scrip. T, 130, 014013

Kamp, I., Tilling, I., Woitke, P., Thi, W.-F., \& Hogerheijde, M. R. 2009, A\&A, in prep.

Meijerink, R., Poelman, D. R., Spaans, M., Tielens, A. G. G. M., \& Glassgold, A. E. 2008, ApJ, 689, L57

Najita, J. R., Doppmann, G. W., Carr, J. S., Graham, J. R., \& Eisner, J. A. 2009, ApJ, 691, 738

Nuth, J. A. 2008, Earth Moon and Planets, 102, 435

Poelman, D. R., \& Spaans, M. 2005, A\&A, 440, 559

Poelman, D. R., \& Spaans, M. 2006, A\&A, 453, 615

Salyk, C., Pontoppidan, K. M., Blake, G. A., et al. 2008, ApJ, 676, L49

Schöier, F. L., van der Tak, F. F. S., van Dishoeck, E. F., \& Black, J. H. 2005, A\&A, 432, 369

Woitke, P., Kamp, I., \& Thi, W.-F. 2009, A\&A, 501, 383 (Paper I) 